Ann. Zootech., 1978, 27 (I), 95-100.

\title{
Note
}

\section{Efficacité comparée des lampes à infra-rouges et des niches thermostatées pour le chauffage d'appoint des porcelets nouveau-nés}

\author{
P. BERBIGIER et J. LE DIVIDICH
}

\author{
Station de Bioclimatologie \\ Centre national de Recherches agronomiques, I.N.R.A., \\ 78000 Versailles (France) \\ Station de Recherches sur l'Élevage des Porcs, \\ Centre national de Recherches zootechniques, I.N.R.A., \\ $7835^{\circ}$ Jouy-en-Josas (France)
}

\section{Résumé}

Sur 3 cycles de mise-bas de I 2 portées chacun, on a comparé l'efficacité de plusieurs modes de chauffage d'appoint des porcelets notıveau-nés : lampe à infra-rouge (traitement témoin) et niches thermostatées à 20,25 et $30^{\circ} \mathrm{C}$. L'efficacité des traitements est estimée d'après la mortalité, les performances de croissance entre la naissance et $2 \mathbf{I}$ jours d'âge et leur attrait pour le porcelet.

Le taux de mortalité post-natale est minimum sous les lampes à I.R. $(9,2$ p. cent); il est respectivement de $I_{5} \mathrm{p}$. cent dans les niches à $30^{\circ} \mathrm{C}$ et de plus de $20 \mathrm{p}$. cent dans les niches régulées à 20 et $25^{\circ} \mathrm{C}$. Il est d' autant plus faible que la fréquentation des aires de chauffage est plus élevée. On ne constate pas de différence significative entre les performances de croissance des animaux soumis aux différents traitements. Néanmoins, l'utilisation des niches à $20^{\circ} \mathrm{C}$ entraîne, à $2 \mathrm{I}$ jours, une diminution du poids moyen des porcelets et du poids total de la portée, de 9 et $19 \mathrm{p}$. cent respectivement par rapport à ceux obtenus avec les niches à $30^{\circ} \mathrm{C}$.

\section{Introduction}

La température de neutralité thermique des porcelets allaités est élevée et très supérieure à celle de leur mère (MOUNT, I968; Kovacs et RAFAI, I973; BERBIgIer, DUSSUel et LE Drvidich, I977). Aussi, pour des raisons physiologiques 
et économiques, essaie-t-on de satisfaire les exigences thermiques du porcelet nouveau-né à l'aide d'un chauffage localisé (lampe à infra-rouge). L'efficacité des dispositifs de chauffage pour assurer un taux de survie élevé et une croissance rapide des porcelets varie cependant avec leur nature et leur puissance (I)E I,A Porte des Vaux et Aumaitre, i967; Adam et Teleki, I972).

L'objet du présent essai est de comparer l'efficacité de deux modes de chauffage d'appoint (lampe à infra-rouge et niches thermostatées à 20,25 et $30^{\circ} \mathrm{C}$ ) estimée d'après la survie et les performances de croissance des porcelets entre la naissance et 1'âge de trois semaines. En outre, le comportement du porcelet vis-à-vis de la source de chaleur est mesuré par le taux de fréquentation de l'aire chauffée. Si l'efficacité des niches s'avère satisfaisante, 1'utilisation de celles-ci, convenablement isolées et thermostatées pourrait permettre une économie d'énergie par rapport au chauffage par lampe à infra-rouge.

\section{Matériel et méthodes}

L'essai a été réalisé entre octobre 1975 et avril 1976 dans une maternité de type danois comportant I 2 cases à sol en béton recouvert d'une litière de paille. Chaque case est divisée en deux parties : l'une réservée à la truie, l'autre aux porcelets dans laquelle sont localisés les dispositifs de chauffage. La truie est maintenue en cage durant la parturition et les premiers jours de lactation, afin de limiter au maximum les pertes par écrasement souvent indépendantes des conditions thermiques du milieu. Un chauffage d'ambiance par air pulsé permet de maintenir la température de $1^{\prime}$ air de la maternité aux environs de 15 à $17^{\circ} \mathrm{C}$.

Pour chaque cycle expérimental de I 2 truies, on constitue avant la nise-bas et en fonction du numéro de portée 4 blocs de 3 truies dont les porcelets sont respectivement soumis aux dispositifs de chauffage d'appoint suivants :

- lampe à infra-rouge (I.R), lot témoin,

- niche à porcelets à $20^{\circ} \mathrm{C}$,

- niche à porcelets à $25^{\circ} \mathrm{C}$,

— niche à porcelets à $30^{\circ} \mathrm{C}$.

Au total, 1'essai a porté sur trois cycles de mise-bas, représentant 36 portées, soit 9 par traitement. Entre chaque cycle expérimental, un vide sanitaire ct une désinfection des locaux ont été effectués.

Les lampes à infra-rouge sont placées à $60 \mathrm{~cm}$ du sol et ont une puissance de $250 \mathrm{~W}$. Les niches (dimensions I $\times 0,8 \times 0,8 \mathrm{~m}$ ), munies, sur leur plus grande face, d'une ouverture carrée de $0,35 \mathrm{~m}$ de côté, ne sont pas éclairées. Le chauffage y est assuré par une plaque chauffante d'une puissance de $400 \mathrm{~W}$, tenant lieu de paroi latérale. La température intérieure est régulée à $\pm I^{\circ} \mathrm{C}$ à l'aide d'un thermostat bilame. Elles sont réalisées en bois et on a constaté que la température résultante sèche, assimilée à celle de la température boule, était toujours égale à la température ambiante à l'intérieur de la niche.

Les porcelets sont pesés individuellement à la naissance, à 7 et à $2 \mathrm{I}$ jours d'âge. Les cas de mortalité sont enregistrés. Deux fois par jour, à heure fixe (o\& et I4 h), on compte pour chaque portée le nombre de porcelets présents dans la zone chauffée. 


\section{Résultats et conclusions}

\section{Taux de mortalité}

Pour l'ensemble de la période de mesure (naissance-2 I j) et indépendamment du dispositif de chauffage d'appoint, on a enregistré un taux moyen de mortalité de I7 p. cent. Celui-ci varie avec le type et l'intensité du chauffage d'appoint : le plus faible taux est obtenu avec les lampes à I.R $(9,2$ p. cent) suivi par les niches à $30^{\circ} \mathrm{C}\left(15 \mathrm{p}\right.$. cent), à $20^{\circ} \mathrm{C}\left(20,5 \mathrm{p}\right.$. cent) et à $25^{\circ} \mathrm{C}(25,5 \mathrm{p}$. cent). L'utilisation des lampes à $I . R$ limite donc au maximum les pertes post-natales. Urıe telle supériorité des lampes par rapport à d'autres dispositifs de chauffage, notam--
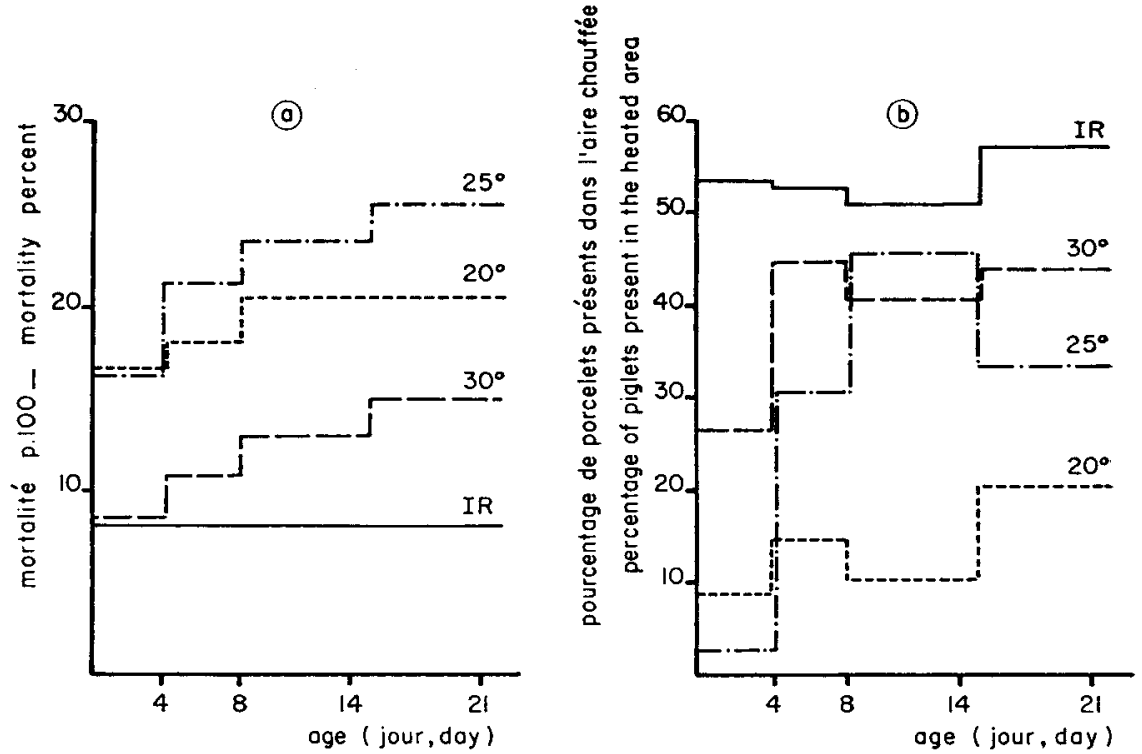

IIG. I.

a) Pertes cumulées (en p. cent de l'cljectif initial) selon le traitement.

Cumulated losses ( $p$. cent of liveborn piglets) according to the treatment.

b) Pourcentage de porcelets dans l'aire chauffée au moment des observations.

Percentage of piglets present in the heated area at the time of the observations.

ment par le sol, a été également rapportée (AumaI'rRe et al., I973). Cependant, les pertes post-natales, obtenues avec les lampes à I.R, paraissent faibles par rapport aux données classiques (BACKSTROM, I973; LEMAN et al., I972). La mortalité est importante surtout durant la période néonatale : 60 à Ioo p. cent des pertes totales surviennent durant les 4 premiers jours de vie (fig. I a), en accord avec les données de KERNkAMP (I965), d'ENGLish (Ig69) et NIIisin et al. (I974).

\section{Comportement des porcelets}

Nos données montrent que le taux de mortalité est in fluencé par le comportement du porcelet vis-à-vis de la source de chaleur. Ainsi (fig. $\mathbf{r}, a$ ) et $b$ )) durant la période néonatale, les lampes à $I . R$ attirent davantage les porcelets que les 
niches. Celles-ci sont d'autant moins fréquentées que leur température intérieure est éloignée de la température de neutralité thermique des animaux (niches à 25 et $20^{\circ} \mathrm{C}$ ). Ces observations encore globales soulignent l'importance de la nature et de l'intensité du chauffage d'appoint sur le comportement du porcelet nouveauné. Celui-ci manifeste une tendance à rechercher le contact maternel notamment durant les 3 premiers jours de vie (TITTERINGTON, I974) correspondant à la période de plus grande sensibilité au froid, ce qui peut favoriser les accidents par écrasement. L'utilisation de lampes à I.R, en attirant davantage le porcelet que les niches, permet de limiter la mortalité néonatale.

\section{Performances pondérales}

Les performances de croissance moyenne des porcelets sont rapportées dans le tableau $I$. En raison de la variabilité des performances, on ne constate pas d'effet significatif des dispositifs de chauffage sur le poids moyen des porcelets

TABI,EAU I

Infuence du mode de chaufiage et de son intensité sur le nombre et lo poids des porcelets (Effect of type and intensity of heating on the number of piglets per litter and weight of piglets)

\begin{tabular}{|c|c|c|c|c|}
\hline \multirow{3}{*}{$\begin{array}{l}\text { Type de chauffage } \\
\text { (Type of heating) }\end{array}$} & \multirow{2}{*}{$\begin{array}{c}\text { Lampe } \\
\text { à infra-rouge } \\
(\text { Infra red } \\
\text { lamp) }\end{array}$} & \multicolumn{3}{|c|}{ Niche à porcelets (piglets' box) } \\
\hline & & $20^{\circ} \mathrm{C}$ & $25^{\circ} \mathrm{C}$ & $30^{\circ} \mathrm{C}$ \\
\hline & $9\left({ }^{(1)}\right.$ & $8\left({ }^{2}\right)$ & 9 & 9 \\
\hline $\begin{array}{l}\text { Nombre moyen de porcelets par portée } \\
\text { (No. of piglets per litter) : } \\
\text { Nés vivants (born alive) } \\
\text { A } 7 \text { jours (at } 7 \text { days of age). } \\
\text { A } 2 \text { I jours (at } 21 \text { days of age) }\end{array}$ & $\begin{array}{l}9,44 \\
8,67 \\
8,67\end{array}$ & $\begin{array}{l}9,75 \\
8,00 \\
7,75\end{array}$ & $\begin{array}{r}10,89 \\
8,55 \\
8,1 \mathrm{I}\end{array}$ & $\begin{array}{r}10,22 \\
9,11 \\
8,67\end{array}$ \\
\hline $\begin{array}{l}\text { Poids des porcelets (kg) (Weight of } \\
\text { piglets }(k g) \text { : } \\
\text { A la naissance (at birth). . . . } \\
\text { A } 7 \text { jours (at } 7 \text { days of age) } \\
\text { A } 2 \text { I jours (at } 2 I \text { days of age). }\end{array}$ & $\begin{array}{l}\mathrm{I}, 25(20)\left({ }^{3}\right) \\
2,44(\mathrm{I} 9) \\
4,98(25)\end{array}$ & $\begin{array}{l}\mathrm{I}, \mathrm{I} 7(2 \mathrm{I}) \\
2,28(22) \\
4,84(2 \mathrm{r})\end{array}$ & $\begin{array}{l}\mathrm{I}, 29(20) \\
2,66(25) \\
5,33(2 \mathrm{I})\end{array}$ & $\begin{array}{ll}I, 30 & (20) \\
2,52 & (21) \\
5,32 & (20)\end{array}$ \\
\hline $\begin{array}{l}\text { Poids total de la portée (kg) (Total } \\
\text { litter weight }(\mathrm{kg}) \text { : } \\
\text { A la naissance (at birth). . . . } \\
\text { A } 7 \text { jours (at } 7 \text { days of age). } \\
\text { A } 2 \mathrm{I} \text { jours (at } 2 \text { I days of age). }\end{array}$ & $\begin{array}{ll}\text { I I, } 86 & (33) \\
2 \text { I, I } & (35) \\
43, \mathrm{I} & (30)\end{array}$ & $\begin{array}{ll}\text { I } 1,44 & (34) \\
\text { I } 8,26 & (33) \\
37,52 & (32)\end{array}$ & $\begin{array}{l}14,08(22) \\
22,27(39) \\
43,24(26)\end{array}$ & $\begin{array}{l}13,25(26) \\
22,94(28) \\
46,10(28)\end{array}$ \\
\hline
\end{tabular}

(') Nombre de portée par traitement. No of litter per treatment.

(2) Une portée dont la truic a été atteinte d'agalaxie a été éliminée. A litter whose mother showed agalactia was eliminated.

( $\left.{ }^{3}\right)$ Coefficient de variation. Coefficient of variation. 
et le poids total de la portée, mais les tendances observées peuvent avoir une très grande importance économique. Ainsi, avec les niches à $20^{\circ} \mathrm{C}$, le poids moyen des porcelets à $2 \mathrm{I}$ jours est inférieur de $9 \mathrm{p}$. cent à celui obtenu dans les niches à $30^{\circ} \mathrm{C}$, l'écart réel étant sans doute sous-estimé en raison des différences d'effectifs. Enfin, pour des tailles de portées identiques, l'emploi des niches à $30^{\circ} \mathrm{C}$ augmente d'environ 6 p. cent (N.S) le poids moyen des porcelets à 2 I jours par rapport au traitement assurant le taux de mortalité le plus faible (lampe à I.R).

En conclusion, le dispositif de chauffage d'appoint par lampes à I.R exerce vis-à-vis des porcelets un attrait immédiat et constant, meilleur que des niches chauffées à 20,25 et $30^{\circ} \mathrm{C}$; il entraîne un taux minimum de mortalité post-natale des porcelets.

Toutefois, l'emploi des niches chauffées à $3^{\circ}{ }^{\circ} \mathrm{C}$, qui permet des performances de croissance des porcelets légèrement supérieures à celles obtenues avec les lampes à I. R, peut être intéressant pour une économie d'énergie à condition d'améliorer leur attirance pour le jeune animal (facilité d'accès, éclairement). De telles observations, confirmées sur des effectifs plus importants d'animaux, peuvent avoir une incidence sur les performances moyennes des truies.

Accepté pour publication en novembre 1977.

\section{Summary}

Comparison between infra-red lamps and thermo-regulated boxes as heating systems for neonate piglets

Using a total of 3 farrowing cycles each including i 2 litters, a comparison was made between the efficiency of heating systems for neonate piglets: infra-red lamps (control treatment) and thermo-regulated boxes $\left(20,25\right.$ and $\left.30^{\circ} \mathrm{C}\right)$. The efficiency of the treatments was estimated. according to mortality, growth performance between birth and $2 \mathrm{I}$ days of age and attractiveness for the piglets.

The post-natal mortality rate was minimum under I.R. lamps ( 9.2 p. cent); it was I 5 p. cent in the boxes heated at $3^{\circ}{ }^{\circ} \mathrm{C}$ and more than $20 \mathrm{p}$. cent in those regulated at 20 and $25 \mathrm{p}$. cent. The longer the piglets stayed in the heated areas the lower was the mortality rate. No significant difference in the growth performance of the animals subjected to the different treatments, was observed. However, use of boxes heated at $20^{\circ} \mathrm{C}$ resulted, at $2 \mathrm{I}$ days, in a 9 and I9 p. cent decrease in the individual mean weight and total litter weight in comparison with the values recorded with the boxes heated at $30^{\circ} \mathrm{C}$ respectively.

\section{Références bibliographiques}

ADAM 'T., TELFKI J., I972. Floor heating and its importance for the pliysiology and performance in piglet rearing. Allattenyésztés, 23, $23-34$.

Aumaitre A., Bichon F., Bina L., Rftragliati J., r973. Importance du milieu environnant (température) dans l'élevage des porcelets : amélioration et contrôle de l'ambiance dans une maternité utilisée sous paille. J. Recherche Porcine en France, I.N.R.A.-I.T.P. Ed. Paris, 239-2 I9.

BACKSTROM I., r973. Environment and animal health in piglet production. A field study of incidences and correlations. Thesis. Acta. Vet. Scand. Suppl., 41; 240 pp.

Berbigrar P., DUSSUfi, A., LE, Dividich J., I977. Variation de température interne et superficielle et confort thermique du nouveau-né. $J$. Recherche Porcine en France, I.N.R.A.I.T.P. Ed. Paris, 29 I-296. 
DE IA POR'́te des VAUX H., Aumaitre A., I967. Influence du milieu thermique sur l'évolution de la température rectale et la glycémie chez le porcelet nouveau-né. Ann. Zootech., 16, $235^{-2} 45$.

ENGLISH P. R., I969. Mortality and variation in growth of piglets. A study of predisposing factors with particular reference to sow and piglet behaviour. Ph. D. Thesis. Univ. Aberdeen.

KERN KAMP H. C. H., 1965. Birth and death statistics on pigs of preweaning age. J.Amer. Vet. Med. Ass., 146, 337-340.

Kovacs F. et RAFAI P., I973. Investigation on the metabolism of new-born and young piglets (Hongrois). Magyar. Ao. Lapja, 28, 531-538.

Leman A. D., Knudson C., Rophrfer H. E., Mueller A. G., I972. Reproductive performance of swine on 76 Illinois farms. J. Amer. Vet. Med. Ass., 161, 1248-1250.

Mounr L. E., 1968. The climatic physiology of the pig. Edward Arnold, London, 27I p.

Nieisen N. C., Christensen K., Bille N., I,ARsen J. I., i974. Preweaning mortality in pigs. I. Herd investigations. Nord. Vet. Med., 26, I37-1 50.

TITTERINGton R. W., I974. The position of the creep heater and the behaviour of piglets during the first week of life. B. S. Agr. Sci. Thesis. Edinburgh School of Agriculture. 